See discussions, stats, and author profiles for this publication at: https://www.researchgate.net/publication/226796487

\title{
Going-down pairs of commutative rings
}

Article in Rendiconti del Circolo Matematico di Palermo · September 2001

DOI: 10.1007/BF02844429

CITATIONS

6

1 author:

Richard Douglas Chatham

Morehead State University

17 PUBLICATIONS 57 CITATIONS

SEE PROFILE

Some of the authors of this publication are also working on these related projects:

Chessboard placement problems View project 


\section{Going-Down Pairs \\ of Commutative Rings}

Doug Chatham

March 16, 2002 


\section{Background}

$G D$ : going-down property (of ring extensions) $I N C$ : incomparability property

$L O$ : lying-over property

$G U$ : going-up property

$\operatorname{Spec}(R)$ : prime ideals of $R$ with the Zariski topology

$\operatorname{Min}(R)$ : minimal prime ideals of $R$

$\operatorname{dim}(R)$ : Krull dimension of $R$

t.d.R $R(T)$ : transcendence degree of the quotient field of $T$ over the quotient field of $R$ 
Def: A ring extension $R \subseteq T$ is open if the contraction map $\operatorname{Spec}(T) \rightarrow \operatorname{Spec}(R)$ given by $P \mapsto P \cap R$ is an open topological map.

Def: Let $\mathcal{P}$ be a property of ring extensions and let $R \subseteq T$ be a ring extension. Then $(R, T)$ is a $\mathcal{P}$-pair if $A \subseteq B$ satisfies $\mathcal{P}$ for all ring extensions $A \subseteq B$ such that $R \subseteq A \subseteq B \subseteq T$.

$L O$-pairs, $G U$-pairs, and $I N C$-pairs have been studied previously in the literature. 
Def: Let $\mathcal{P}$ be a property of ring extensions. A domain $R$ is a $\mathcal{P}$-domain if for all overrings $S$ of $R, R \subseteq S$ satisfies $\mathcal{P}$.

Ex: If a domain $R$ is Prüfer or has $\operatorname{dim}(R) \leq 1$, then $R$ is a $G D$-domain.

Ex: Let $R$ be a Noetherian domain. Then $R$ and all its overrings are open-domains $\Longleftrightarrow R$ is semilocal and $\operatorname{dim}(R) \leq 1$ (Papick, 1976). 
Def: We say $(R, T)$ is a $\mathcal{P}$-domain pair if $S$ is a $\mathcal{P}$-domain, for all $S$ such that $R \subseteq S \subseteq T$.

Ex: If $R$ is a Prüfer domain and $K$ is its quotient field, then $(R, K)$ is a $G D$-domain pair.

Ex: If $k \subseteq K$ is an algebraic field extension, then $(k, K)$ is an open-domain pair.

Ex: Let $R$ be Noetherian and $k$ the quotient field of $R$. Then $(R, k)$ is an open domain pair $\Longleftrightarrow R$ is semilocal and $\operatorname{dim}(R) \leq 1$. (Restatement of above example of Papick) 
Def: A ring $R$ is a $\mathcal{P}$-ring if $\forall P \in \operatorname{Spec}(R), R / P$ is a $\mathcal{P}$-domain. (For $\mathcal{P}=G D$, Dobbs 1996)

Ex: $\operatorname{dim}(R) \leq 1 \Rightarrow R$ is a $G D$-ring. Other examples of $G D$-rings include pseudo-valuation rings, chained rings, and finite products of $G D$ rings.

If $R$ is a domain, then $R$ is a GD-ring (resp., open-ring) $\Longleftrightarrow R$ is a $G D$-domain (open domain). 
Def: We say $(R, T)$ is a $\mathcal{P}$-ring pair if $S$ is a $\mathcal{P}$-ring, $\forall S \ni R \subseteq S \subseteq T$.

Proposition 1 The following are equivalent:

1. $(R, T)$ is a GD-ring pair (resp., open-ring pair);

2. For each $Q \in \operatorname{Spec}(T)$, $(R /(Q \cap R), T / Q)$ is a $G D$-domain pair (open domain pair);

3. For each $Q \in \operatorname{Min}(T)$,

$(R /(Q \cap R), T / Q)$ is a $G D$-domain pair (open domain pair). 


\section{Transcendence degree results}

Lemma 2 If $k$ is a field contained in a domain $T$, then $(k, T)$ is a $G D$-domain pair $\Longleftrightarrow$ t.d.k$(T) \leq 1$.

Theorem 3 If $\operatorname{dim}(R)=0$, then the following are equivalent:

1. $(R, T)$ is a $G D$-ring pair;

2. $t . d_{R / P \cap R}(T / P) \leq 1, \forall P \in \operatorname{Spec}(T)$;

3. $t . d \cdot R / P \cap R(T / P) \leq 1, \forall P \in \operatorname{Min}(T)$. 
Lemma 4 If $k$ is a field contained in a domain $T$, then $(k, T)$ is an open-ring pair $\Longleftrightarrow k \subseteq T$ is an algebraic field extension.

Theorem 5 If $\operatorname{dim}(R)=0$, then the following are equivalent:

1. $(R, T)$ is a open-ring pair;

2. $t . d \cdot R / P \cap R(T / P)=0, \forall P \in \operatorname{Spec}(T)$;

3. $t . d_{R / P \cap R}(T / P)=0, \forall P \in \operatorname{Min}(T)$;

4. $(R, T)$ is an INC-pair;

5. $T$ is an integral extension of $R$. 


\section{What if $\operatorname{dim}(R) \geq 1$ ?}

Proposition $6 R[X]$ is a $G D$-ring $\Longleftrightarrow$ $\operatorname{dim}(R)=0$.

Corollary 7 If $(R, T)$ is a GD-ring pair and $\operatorname{dim}(R)>0$, then $R \subseteq T$ is an algebraic ring extension.

The converse of this corollary fails: consider $(\mathbb{Z}[X], \mathbb{Z}[X][\sqrt{2}])$.

$G D$-ring pairs need not be $I N C$-pairs, even when $\operatorname{dim}(R)>0$.

Proposition 8 If $\operatorname{dim}(R) \leq 1$ and $(R, T)$ is an INC-pair, then $(R, T)$ is a GD-ring pair.

For $\operatorname{dim}(R) \geq 2$, INC-pairs need not be $G D$ ring pairs. 
Proposition 9 If $(R, T)$ is an open-ring pair, then $(R, T)$ is an INC-pair.

Proposition 10 Let $(R, T)$ be a normal pair of domains (i.e. $A$ is integrally closed in $B, \forall R \subseteq$ $A \subseteq B \subseteq T)$. Then $(R, T)$ is a $G D$-domain pair (resp., open domain pair) $\Longleftrightarrow R$ is a $G D$ domain (open domain). 


\section{Relation between $\mathcal{P}$-ring pairs and $\mathcal{P}$-pairs}

If $R$ is a $G D$-domain, $R \subseteq T$ satisfies $G D$ for all domains $T$ containing $R$. (Dobbs-Papick, 1976)

So $(R, T) G D$-domain pair $\Rightarrow(R, T) G D$-pair.

If $R$ is a open domain, $R \subseteq T$ is open for all domains $T$ containing $R$. (Papick, 1976)

So $(R, T)$ open domain pair $\Rightarrow(R, T)$ open pair. 
Proposition $11\left(\mathbb{F}_{p}, \mathbb{F}_{p}(X)\right)$ is an INC-domain pair that is not an INC-pair.

Also, $R$ a $G D$-ring does not imply $R \subseteq T$ satisfies $G D$ for all rings $T$ containing $R$. (Dobbs, 1996)

Theorem 12 Let $\mathcal{P} \in\{G D$, open $\}$. Then $R$ a $\mathcal{P}$-ring $\Rightarrow R \subseteq T$ satisfies $\mathcal{P}$ for all $T$ with a unique minimal prime.

Corollary 13 Let $\mathcal{P} \in\{G D$, open $\}$. If $T$ is a ring with a unique minimal prime and $(R, T)$ is a $\mathcal{P}$-ring pair, then $(R, T)$ is a $\mathcal{P}$-pair. 


\section{Are $\mathcal{P}$-pairs always $\mathcal{P}$-ring pairs?}

Ex: Let $k \subseteq K$ be an algebraic field extension and $T$ a ring that is not a $G D$-ring. Then $(k \times T, K \times T)$ is a $G D$-pair and an open-pair that is not a $G D$-ring pair (or an open-ring pair).

Ex: Let $L \subset K$ be an algebraic extension of distinct fields. Let $T=K[[X, Y]]$ and $R=$ $L+X T+Y T$. Then $(R, T)$ is a $G D$-pair of domains that is not a $G D$-domain pair. 


\section{Flat pairs and flat-domain pairs}

Def: A ring extension $R \subseteq T$ is flat if $T$ is a flat $R$-module.

A domain $R$ is a flat-domain $\Longleftrightarrow R$ is a Prüfer domain (Richman, 1965)

Therefore, we will refer to a flat-domain pair as a "Prüfer domain pair." 
Proposition 14 Let $R$ be a domain contained in a field $K$. Then the following are equivalent:

1. $(R, K)$ is a flat pair;

2. $(R, K)$ is a Prüfer domain pair;

3. either

(a) $R \subseteq K$ is an algebraic field extension; or

(b) $R$ is a Prüfer domain and $K$ is the quotient field of $R$. 
Proposition 15 Let $R \subseteq T$ be domains with $R$ Prüfer. Then the following are equivalent:

1. $(R, T)$ is a flat pair;

2. $(R, T)$ is a Prüfer domain pair;

3. either

(a) $R \subseteq T$ is an algebraic field extension; or (b) $T$ is an overring of $R$. 